\author{
Krzysztof Sakowski*
}

\title{
FREMDES ODER EIGENES IN DER SATZSTRUKTUR? \\ - ZU EINIGEN KOGNITIVEN ASPEKTEN \\ DER VERBALKLAMMER IM DEUTSCHEN
}

\section{Einleitung und Problemstellung}

Die Verbalklammer gehört zu solchen Phänomenen der deutschen Sprache, die den Lernenden mit Sicherheit die meiste Aufmerksamkeit sowohl beim Verstehen als auch bei der Produktion abverlangen. Es ist keineswegs die einzige Sprache, bei der sich diese Art der Informationsstrukturierung herausgebildet hat, aber es ist die einzige große Sprache, in der die Verbalklammer in diesem Umfang vorkommt. Es ist daher vom Interesse, aus welchen Gründen sich eine entfernende Satzstrukturierung so erfolgreich durchgesetzt hat und ob sie als Resultat von Einwirkungen anderer Sprachkulturen zu verstehen ist oder eher schon in der Geburtsstunde des modernen Deutschen unter möglichen Varianten vorhanden war. Allerdings will ich im Rahmen dieses Beitrags nicht nur auf die rein strukturellen Eigenschaften einen Blick werfen, sondern vor allem der Motivation der Verbaltrennung auf die Spur kommen und sie aus dem kognitiven Blickwinkel beleuchten.

\section{Herkömmliche Erkenntnisse zum Vorkommen der Verbalklammer im Deutschen}

Wie es von Admoni (1982: 302) und Eroms (2000: 133) betont wird, übt die Verbalklammer in Bezug auf die Entfaltung der semantischen Information im Hauptsatz folgende Funktionen aus:

1. Sie bildet ein Gerüst, innerhalb dessen die gesamten Informationen eingerahmt werden können. Die Entfaltung dieser Information innerhalb des Rahmens richtet sich nach dem Prinzip des steigenden Mitteilungswertes. (Thema-Rhema).

* Dr. Krzysztof Sakowski, Universität Łódź, Institut für Germanische Philologie, Pomorska 171/173, 90-236 Łódź. E-Mail: krzysztofsakowski@o2.pl 
2. Die semantische, innere Struktur der Verbalklammer entspricht dem Prinzip des steigenden Mitteilungswertes. Somit wird gewährleistet, dass die informative Spannung des Hörers nicht nachlässt.

3. Aus den beiden genannten Funktionen lässt sich herleiten, dass die Verbalklammer an sich nicht nur eine thematisch-rhematische Struktur ist, sondern in erster Linie, dass ihr ans Ende gesetztes Zweitglied das am stärksten rhematisierte Element des Satzes ist.

Die Herausbildung der Satzklammer und daraufhin auch der Verbalklammer im Deutschen wird in der einschlägigen Literatur oft als eine rein stilistische Tendenz dargestellt. Viele traditionelle Grammatiken betrachten diese Entwicklung als ein Element des stilistischen Fortschritts und nicht als eine grammatische Bedingung. Besonders interessant scheinen in dieser Hinsicht die Feststellungen, dass bestimmte soziokulturelle Faktoren für diesen Sachverhalt verantwortlich gemacht werden, wie z.B. das Vorbild des Humanistenlatein (vgl. mit Behagel 1892), der Einfluss der Schulgrammatik (vgl. mit Biener 1922) sowie die Verbreitung des Kanzleistils (vgl. mit Ebert 1986). Oft wird in den Studien zu dieser Thematik argumentiert, dass der Wandel von fakultativer zur obligatorisch auszuführender Klammerstellung im Deutschen keine kategoriale oder grammatische Ursachen haben kann, da es auch nach Lenerz (1984) bestimmte Varianten der Ausklammerung gibt.

Die Verbalklammer ist demzufolge ein Resultat verschiedener Faktoren, die zum heutigen Zustand in der Wortstellung und darunter natürlich auch in der Herausbildung der Verbalklammer im Deutschen geführt haben. Sie lassen sich nur aus einer weiten geschichtlichen Perspektive ergründen. Die Satzgliedstellung nach den gleichen noch heute geltenden Anordnungsprinzipien hat sich schon im Althochdeutschen herausgebildet, obwohl damals noch eine gewisse Freiheit in der Anordnung der Satzglieder bestand. Aus den vorhandenen Belegen (Ebert 1986: 131) geht jedoch eindeutig hervor, dass Ansätze zur verbalen Klammer bereits damals vorhanden waren. Im Laufe der Zeit hat sich die Verbalklammer als Satzstruktur durchgesetzt, wo das 17. Jh. als die Epoche der höchsten Entfaltung der Rahmenkonstruktion gelten kann.

Eine erkennbare Tendenz zur Klammerbildung war also schon auf sehr frühen Etappen in der Entwicklung der deutschen Sprache vorhanden und bildete somit in meiner Erkenntnis eine essenzielle Grundlage in der Prägung der kognitiven Wahrnehmungswege der folgenden Generationen der deutschen Sprachgemeinde. Die gemeinsamen Wurzeln für diese Grundlage lassen sich allerdings auch heute noch in den anderen germanischen Sprachen finden. Ich will deswegen im Weiteren auf dieses Phänomens mit Hilfe unterschiedlicher Methodologien: der historisch-diachronen sowie der synchron-kontrastiven eingehen und somit die verbale Klammerbildung aus einer weiteren Sicht erfassen. Damit will ich ausdrücklich zeigen, dass der Klammerbildung nicht nur eine rein stilistische Dimension, wie es postuliert wird, beigemessen werden soll, 
sondern es liegt ihr auch vor allem ein semantisches Prinzip zugrunde, wonach man sich in Einzelfällen im heutigen Deutsch noch richtet. Darauf will ich im Rahmen dieses Beitrags auch im Weiteren eingehen.

\section{Diachrone und kontrastive Perspektive der Klammerbildung}

Es ist eine auffallende Tatsache, dass in allen germanischen Sprachen derzeit Konstruktionen vorhanden sind, die aus einem Verb und einer zusätzlichen Partikel bestehen (ich übernehme hierzu die Benennung aus dem Englischen: verb-particle constructions $)^{1}$. Alle diese Konstruktionen werden nach einer semantischen und zwei voneinander unabhängigen syntaktischen Regel gebildet. Dazu einige Beispiele aus den zitierten Quellen mit eigener Übersetzung:

1) Schwedisch (nach Braunmüller 1999: 65)

Vi målade över tapeten med grön färg.

(Wir strichen die Tapeten mit grüner Farbe über.)

2) Dänisch (nach Braunmüller 1999: 65)

Vi malede tapetet over med grøn farve.

(Wir strichen die Tapeten mit grüner Farbe über.)

3) Norwegisch (nach Askedal 1994: 262)

Boka kjem ut i neste veke.

(Das Buch kommt nächste Woche aus.)

4) Englisch

The book comes out next week

(Das Buch kommt nächste Woche (he)raus.)

5) Isländisch (nach Thráinsson 1994: 175)

Fjöldi manns tók bœkurnar fram.

(Viele Menschen nahmen die Bücher aus.)

6) Niederländisch (Booij 2002: 21)

Hans belde zijn moeder op.

(Hans rief seine Mutter an.)

${ }^{1}$ Die in den 70-er und 80-er Jahren noch gängige Einteilung in verbale Halbpräfixe und Präfixoide ist auf heftige Kritik, besonders in der englischsprachigen Sprachwissenschaft gestoßen (vgl. z.B. Schaeder 1997: 193). Es wurde vorgeworfen, dass diese Einteilung nicht vollständig den ganzen morphologisch denkbaren Bereich deckt (wie z.B. kennenlernen, brustschwimmen) sowie einige Mischformen außer Acht lässt (abverlangen). Um die terminologische Unsicherheit zu vermeiden, greife ich im Rahmen dieses Beitrags zur Bezeichnung: Verbalpartikel. 
Die besagte semantische Regel, die bei allen gezeigten Beispielen gleich ist, lautet: die Verbalpartikel hat den semantischen Wert des Verbs um ein zusätzliches Element zu ergänzen. Dieses Element kann entweder eine reale Handlung darstellen, bei der das Landmark (im Sinne von Langacker 1987) im Vergleich zum Basisverb modifiziert verändert wird (streichen vs. überstreichen), so dass die Beziehungen der realen Welt wiedergeben werden, oder eine konzeptuelle Metapher bilden, um die Größenrelation zur realen Handlung virtuell nachzubilden (herauskommen), oder aber eine konzeptuelle Metapher sein, die im Laufe der Zeit gefestigt wird und wie eine idiomatische Wendung verwendet wird, (anrufen), ohne dass man die Entstehungsmotivation für diese Metapher aus der heutigen Sicht zurückverfolgen kann. Hauptsächlich beruht aber die Zugabe einer Partikel zum Verb auf Modifizierung seiner Hauptbedeutung in unterschiedlichem Ausmaß, so dass sein semantischer Status (realitätsbezogen, konzeptuell metaphorisch oder idiomatisch) durch diese Operation variiert wird.

Syntaktisch gesehen haben wir es entweder mit einer entfernenden Stellung der Verbalpartikel (Deutsch, Niederländisch und Isländisch) oder mit der Stellung gleich nach dem Kernverb (Englisch, Dänisch, Norwegisch und Schwedisch) zu tun. Nichtsdestoweniger können innerhalb einer Sprache beide Regeln gemischt auftreten, wofür Englisch als Hauptbeispiel gelten kann:

7) I'll look the answer right up.

8) Please shut the gas completely off.

Diese unterschiedlich zu markierende Distribution kann auch für andere Konstruktionen festgestellt werden, was eindeutig auf die Satzklammer- und nicht die Verbklammerperspektive hinweist.

9) This has without doubt been a very good debate.

10) The man John was given the present by.

Ich will hier allerdings die formalen Vorgaben für die eine oder andere Verbalpartikelstellung im Englischen nicht diskutieren, sondern nur das strukturelle Potential verdeutlichen, dass die Klammerfähigkeit nicht vollständig aufgehoben ist.

Die Tendenz zur Poststellung der Verbalpartikel im Englischen bei den heutigen phrasal verbs hat sich jedoch relativ erst spät herausgebildet, d.h. in den letzten 300-400 Jahren. Im Altenglischen (5 Jh. bis zum $12 \mathrm{Jh}$.) wurden die Verbalpartikeln noch nicht trennbar als Präfixe dem Kernverb angehängt. ${ }^{2}$ Millward (1996) berichtet von nicht separierbaren Verbalpartikeln, als Ursprungsformen der heutigen phrasal verbs, die im Altenglischen auftauchen. Als Beispiel nennt

\footnotetext{
${ }^{2}$ Beleg vom 25.06.2016, erhältlich unter http://www.englishexperts.com.br/the-origin-of-thephrasal-verb/
} 
er die Opposition von baernan (im heutigen Englisch to bum) and fbrbaernan (im heutigen Englisch to burn up). Denison (1993: 5) beruft sich in seiner Arbeit auf das Beispiel beraedan (to dispossess) als Opposition zu raedan (to advise). In der neueren Phase des modernen Englischen (15 Jh. bis zum $17 \mathrm{Jh}$. $)^{3} \mathrm{kam}$ es zu einer explosionsartigen Entwicklung dieser Struktur wie auch zur Trennung der Verbalpartikel und ihrer Poststellung in der Satzstruktur. Daraus ergibt sich das Bild einer stark ausgeprägten Neigung zur analytischen Satzordnung.

Im Althochdeutschen bestehen hingegen schon von frühen Entwicklungsphasen an zahlreiche Beweise für verbale Trennbarkeit und Distanzhaltung (nach Bock, 2009: 31) z.B. $\hat{u}$-springan ( $\hat{u} f>$ auf) "aufspringen ", $\hat{f}$-stantan "aufstehen", $\hat{u f}$-gangan (îf>aus) "ausgehen", "hinausgehen", zuo-gangan "auf jdn. zukommen", "sich nähern", nidar-stîgan "niedersteigen". Die ahd. Satzgliedstellung war jedoch, wie schon im vorherigen Kapitel angesprochen, in allen Satzarten grundsätzlich noch relativ frei und entschied nicht, wo genau die abgetrennte Verbalpartikel zu stehen hat. Der Trend zur Absonderung der Verbalpartikel lässt sich jedoch schon in dieser Zeit beobachten. Einen anderen Beweis für das frühe Vorkommen des analytischen Satzbaus findet man bei der neuerdings erschienenen Tatian-Übersetzung ${ }^{4}$, die um 850 datiert wird.

\section{1) thaz gibrieuit uuvrdi al these umbiunerft $(\mathrm{T} 35,9)$ dass aufgelistet würde all diese Menschheit \\ 12) thaz in mir habet sibba $(\mathrm{T} 290,8)$ damit in mir habt Frieden}

Aus den gezeigten Fragmenten, die naturgemäß keinen Anspruch auf Vollständigkeit erheben aber dennoch wichtige Ansatzpunkte verdeutlichen, gehen zwei belangreiche Tatsachen hervor:

1. die Ausprägung zur analytischen Handhabung der Verbalkomplexe ist eine in den germanischen Sprachen weit verbreitete Präferenz;

2. die Verbalklammer ist ein besonderes Ausdrucksmittel des analytischen Satzbaus, das nicht nur für Deutsch spezifisch ist sondern auch für andere germanische Sprachen nachgewiesen werden kann.

\section{Hypothese}

Ich gehe hier von der methodologischen Annahme aus, die in der kognitiv basierten Linguistik eine fundamentale Größe bildet, dass grammatische Struktur einer natürlichen Sprache immer einen sekundären Charakter aufweist und eine

\footnotetext{
3 Ebenda.

${ }^{4}$ Gefunden am 25.06.2016. unter http://titus.uni-frankfurt.de/texte/etcs/germ/ahd/tatian/tatia.htm,
} 
bestimmte semantische Funktion implementieren soll. Die Grammatik besteht aus bestimmten Gruppierungen von Elementen symbolischer Natur, deren Entstehung eine Frage der Graduierung, der Intensität ist. Grammatische Strukturen reflektieren demnach das Zusammenspiel zwischen der diskursiven und der deskriptiven Ebene (Langacker 1991, 1997).

Strukturen jeder kognitiven Natur, auch sprachlicher, entstehen durch Induktion. Sie sind das Ergebnis der Beobachtung eines Details oder vieler Details, bis ein Schema sich merklich abzeichnet, das sich im Rahmen der gegebenen Abstraktionsprinzipien erst als besonders und im Laufe der Zeit als normal, typisch für diese Gruppierung klassifizieren lässt. Grammatische Strukturen verkörpern demzufolge eine Art vorprogrammiertes Wissen mit operativem Charakter (Wilss 1992: 230). Darauf bezieht sich eine Reihe der Theorien im Bereich der Konstruktionsgrammatik. ${ }^{5}$ Die gesamte Grammatik einer Sprache besteht demnach aus taxonomischen Hierarchien solcher Einheiten.

Diese Theorie weist also unmittelbar auf einen quasi quantitativen Charakter der Regelbildung bei grammatischen Strukturen hin. Allerdings spielt auch bei der Verarbeitung der Neubildungen die Analogie eine tragende Rolle. Bemerkenswert ist hierbei, dass die Analogien hauptsächlich auf phonologischer Ebene hergestellt werden. Dies lässt sich auch anhand der Ergebnisse neuerer Forschungen der letzteren Jahre im Syntaxbereich sehr gut nachvollziehen. Kashak und Glenberg (2000) haben beispielsweise experimentell nachgewiesen, dass das strukturelle Muster einer Konstruktion nur eine allgemeine Szene bilden kann, während erst die Angabe bestimmter funktionaler Elemente Details hervorbringen kann, die auf phonologisch analoge Art und Weise mit metaphorisch benachbarten Phänomenen realisiert werden. Goldberg (2009: 94) weist dazu darauf hin, dass die Bestandteile einer grammatischen oder festen semantischen Struktur immer Paarungen von Form und Bedeutung bleiben, so dass sie durch Akte sprachlicher Kreation von ihrer ursprünglichen Rolle abgelöst werden können.

Aus der Perspektive des kognitiven Forschungsparadigmas kann das Phänomen der deutschen bzw. höher gegriffen, germanischen Verbalklammer und daraufhin der Regeln zur verbalen Trennbarkeit vs. Untrennbarkeit somit in ein etwas anderes Licht gestellt werden. Wie ich am Anfang dieses Aufsatzes mit zahlreichen Beispielen verdeutlicht habe, übt die Klammerfähigkeit als strukturelle Eigenschaft der deutschen Sprache einen erheblichen Einfluss auf die Satzfolge aus. Dieser Einfluss ist allerdings weiter zu definieren als nur in Bezug auf die verbale bzw. adverbiale Trennbarkeit und lässt sich beobachten, wenn man andere Klammertypen mit einbezieht, bei denen die von uns angesprochene Trennbarkeit überhaupt keine Rolle spielt, nämlich bei der Nominalklammer. Auch hier kann man die Tendenz zum Fernhalten der zusammengehörenden Informationen bemerken, wie etwa:

\footnotetext{
${ }^{5}$ Siehe dazu: Hilpert (2014), Goldberg (2009).
} 


\section{3) Die neu eingeführte, aber bereits umstrittene Regelung.}

Die Klammerbildung an sich deutet also in erster Linie auf den Bedarf hin, einen Sonderplatz jener semantischen Einheit anzubieten, die im Hinblick aufs Ganze hervorgehoben werden soll. Übertragen wir diesen Bedarf auf die strukturelle Trennungseigenschaft des Verbalpartikels, so bemerken wir auch die Notwendigkeit einer gezielten Akzentuierung, also Setzung des höher gesetzten Mitteilungswerts. Prozessual gesehen kommt es zur Isolierung des Verbalpartikels im Rahmen des Grounding-Prozesses, d.h. während der kontextuellen Anpassung an den Satz- bzw. Textinhalt, was wiederum auf die semantische Relevanz dieses Teils hinweist. Mit anderen Worten wird die Verbalpartikel, genauso wie auch die zum Vergleich gestellten nonverbalen Elemente, getrennt gehalten, um ihre Bedeutung zu betonen.

Wenn wir also unsere früheren Auseinandersetzungen im diachron-kontrastiven Teil dieses Aufsatzes erneut anführen und die daraus resultierenden Schlussfolgerungen mit dieser Hypothese in Verbindung setzen, so ist die Tendenz zum Fernhalten und zur Trennung der Verbalteile überhaupt ein Ausdruck für das Bedürfnis nach semantischer Quantitäts- oder Graduitätsmarkierung. Dieses Bedürfnis kann deswegen ein direkter Auslöser des analytischen Satzbaus sein (siehe hierzu auch Levin-Steinmann, 2004: 109-111).

\section{Beweis}

Einen besonderen Fall bilden in dieser Hinsicht die Verben mit der Verbalpartikel durch, die zwei Deutungsvarianten zulassen und sowohl als trennbar als auch als untrennbar vorkommen können. Diese Schwankung resultiert aus dem Charakter des oben dargestellten Prozessverlaufs des Groundings. Wird das Ende, bzw. die Richtungsangabe des Prozesses wichtiger als der semantisierte Prozess selbst, kommt es zur symbolischen Trennung des dafür stehenden Teils, um es als wichtiger anzudeuten. Wir können von einer zweistufigen Bedeutungsstruktur der Verben mit einer Verbalpartikel sprechen. Die erste Stufe determiniert den Prozess im Allgemeinen, d.h. definiert den Prozess als eine Größe ohne den Charakter dieses Prozesses im Detail anzugeben. Die zweite Stufe ermöglicht eine Entscheidung zu treffen, welches dieser Bestandteile eine ausschlaggebende Rolle für seine genaue semantische Profilierung spielt und dieses Element entsprechend betont.

durchkreuzen sie durchkreuzte Amerika

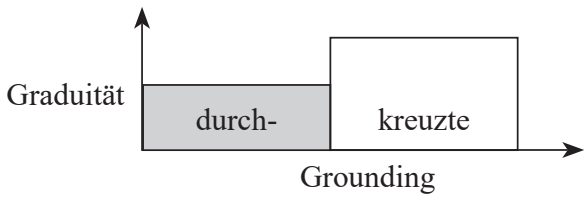


sie kreuzte den Brief durch

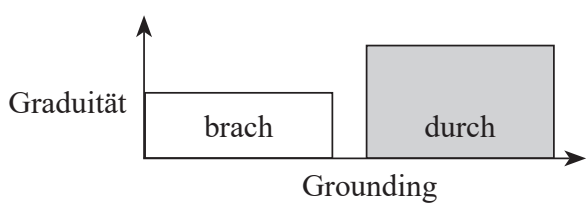

\section{durchfallen \\ der Stein durchfiel die Strecke \\ in einer Sekunde}

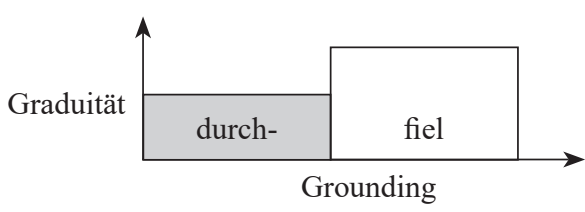

die kleinen Steine fielen

[durch die Öffnung] durch

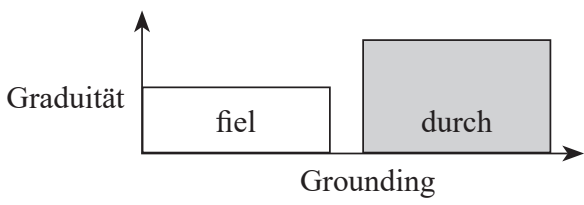

\section{durchbrechen \\ er durchbrach seine Arbeit}

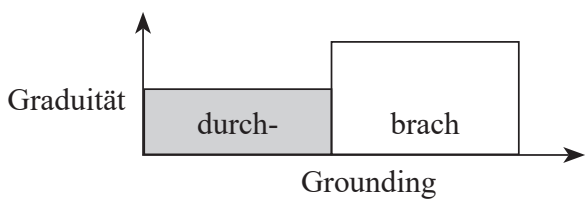

er brach das Brett durch

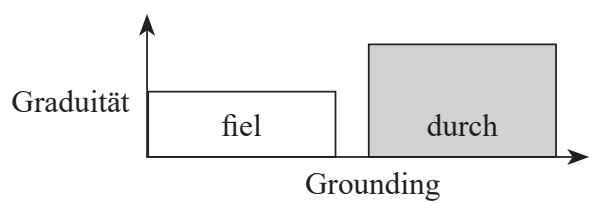

Daraus resultiert auch das unterschiedlich zu lösende, prosodische Distributionparadigma. Je nach Bedarf zum Hervorheben der spatialen (räumlichen) Relevanz wird also das Erstglied entweder akzentuiert (seine spatiale Bedeutung betont) oder ausgeblendet (in der Prozessbeschreibung anwesend aber spatial nicht entscheidend). Die didaktisierende Klassifizierung dieser Verben nach metaphorischer bzw. wörtlich zu nehmender Bedeutung kann sich deshalb nicht als geltend erweisen, zumal viele Ausnahmen diese Zuschreibung zunichtemachen. Die spatiale Markierung der Struktur kann nämlich auch bei metaphorischer Perspektive eine entscheidende Rolle spielen. Hierzu einige Beispiele zur Veranschaulichng: 
14) Ich habe die Sache noch einmal durchgedacht.

15) Der Plan ist wirklich gut durchdacht.

Die spatiale Markierung signalisiert in (14) einen erneuten Versuch gedanklich an ein Problem heranzukommen, während in (15) dieser Signal semantisch nicht relevant erscheint.

16) Das Flugzeug hat die Schallmauer durchbrochen.

17) Sein Hass ist durchgebrochen.

Und wieder macht sich die Tendenz zur Hervorhebung des jeweiligen verbalen Elements durch strukturelle Markierung je nach semantischem Bedarf deutlich. In 16 ist das Brechen, also der prozessuale Teil relevant und nicht die räumliche Orientierung. In (17) ist das plötzliche Zutagekommen der Gefühle wichtiger als der Prozess selbst, d.h. dass das spatiale Charakter betont werden muss.

18) Er hat die Schuhe durchgelaufen.

19) Das Projekt hat viele Stadien durchlaufen.

Dieselbe Regelhaftigkeit macht sich auch im letzen Beispielpaar bemerkbar. Bei (18) ist die Bedeutung von durch wichtiger als das Betonen der Tätigkeit selbst. Umgekehrt in (19), wo der lange Weg des Projekts angedeutet werden soll.

\section{Zusammenfassung}

Die Verbalklammer dient zum Ausdruck der kommunikativen Funktion in der Sprache, wodurch dem Hörer ein Gefüge angeboten wird, damit er die Information auf eine vorher definierte Art und Weise dekodieren kann. Dieses Gefüge besteht aus gewissen Anhaltspunkten, die als Signale für das Verstehen der Information dienen. Nicht nur das Auftreten dieser Signale, sondern auch ihre Position im Satz helfen bei der Bearbeitung der darin enthaltenen Information. Die Klammerfähigkeit ist daher eine kommunikativ bestimmte Größe, die als Oberstruktur eine der Hauptrollen in der deutschen Syntax spielt. Solche oder ähnliche Strukturen können jedoch auch in mehreren anderen germanischen Sprachen vorgefunden werden, wie beispielsweise im Niederländischen oder Isländischen. Ihre Wurzeln greifen weit in die urgermanische Zeit. Die Verbalklammer kann aus diesem Grund als ureigene Eigenschaft des Deutschen genannt werden, was auch die diachronen Untersuchungen bewiesen haben.

Die heutige Strukturentwicklung der Verbalklammer resultiert aus der Tendenz, den Relevanzgrad der Information zu strukturieren und zu differenzieren. Dieser Bedarf an Graduierung liegt der analytischen Handhabung der Informa- 
tionsabstufung zugrunde, was die Wichtigkeit dieser profilierenden Information (Verbalpartikel) betont. Interessant klingt in dieser Hinsicht, dass das Deutsche analytisch nur einen Teil der Verbalpartikelgruppe als relevant betrachtet. Es sind nur spatial bedeutsame Relationen (wörtlich, metaphorisch oder sogar idiomatisch verstanden). Diese These müsste dennoch in einer umfassenderen Untersuchung verifiziert werden.

\section{LITERATURVERZEICHNIS}

Admoni W. (1982), Der deutsche Sprachbau. München: Beck.

Askedal J. O. (1994), Norwegian. In: König E., van der Auwera J. (Hrsg.), The Germanic Languages, 219-270. London: Routledge.

Bauer G. (2001), Einführung in die Diachrone Sprachwissenschaft. Göppingen: Kümmerle Verlag. Behagel O. (1891), Geschichte der deutschen Sprache. In: Hermann P. (Hrsg.), Grundriss der germanischen Philologie I, 526-633. Strassburg: Trübner.

Booij G. (2002), The Morphology of Dutch. Oxford.

Braunmüller K. (1999), Die skandinavischen Sprachen im Überblick. Tübingen: Francke.

Dittmer A., Dittmer E. (1998), Studien zur Wortstellung - Satzgliedstellung in der althochdeutschen Tatianübersetzung, Göttingen.

Denison D. (1992), The Origins of Completive up in English. Neuphilologische Mitteilungen. Berlin.

Donalies E. (2005), Die Wortbildung des Deutschen. Tübingen: Gunter Narr Verlag.

Ebert R. P. (1986), Historische Syntax des Deutschen II. 1300-1750. 2. überarbeitete Auflage 1999. Berlin.

Eroms H. (2000), Syntax der deutschen Sprache. Berlin-New York:Walter de Gruyter.

Feldman J. A. (2006), From molecule to metaphor. Cambridge, MA: MIT Press.

Goldberg A. (2009), The Nature of Generalization in Language. In: Cognitive Linguistics 20/1: 93-127.

Hilpert M. (2014), Construction Grammar and its Application to English. Edinburgh: Edinburgh University Press.

Lenerz J. (1984), Syntaktischer Wandel und Grammatiktheorie. Tübingen.

Kaschak M., Glenberg A. (2000), Constructing meaning: The role of affordances and grammatical constructions in sentence comprehension. In: Journal of Memory \& Language, 43, 508-529.

Levin-Steinmann A. (2004), Kognitive Aspekte des Analytismus am Beispiel der Funktionsverbgefüge. In: Hinrichs U. (Hrsg.), Die europäischen Sprachen auf dem Wege zum analytischen Sprachtyp, 103-126. Otto Harassowitz: Wiesbaden.

Langacker R. W. (1991): Concept, Image, and Symbol: The Cognitive Basis of Grammar. BerlinNew York: Walter de Gruyter.

Langacker R. W. (1997), Consciousness, Construal, and Subjectivity. In: Stamenov M. (Hrsg.), Language Structure, Discourse and the Access to Consciousness, 49-75. Amsterdam \& Philadelphia: John Benjamins.

Millaward C. M. (1996): A Biography of the English Language. 2nd edition. Fort Worth: Harcourt Brace.

Schaeder B. (1997), Getrennt- und Zusammenschreibung. In: Augst G., Blüml K., Nerius D., Sitta H. (Hrsg.), Zur Neuregelung der deutschen Orthographie: Begründung und Kritik: Begrundung Und Kritik (Reihe: Germanistische Linguistik, Band 179), 157-201. Tübingen: Niemeyer.

Thráinsson H. (1994), Icelandic. In: König E., van der Auwera J. (Hrsg.), The Germanic Languages, 142-189. London: Routledge. 
Willis S. L. (1992), Cognition and everyday competence. In: Schaie K. W., Lawton M. P. (Hrsg.), Annual review of gerontology and geriatrics, Vol. 11, 80-109. New York: Springer

Ziem A., Lasch A. (2013), Konstruktionsgrammatik. Konzepte und Grundlagen gebrauchsbasierter Ansätze. Dresden: de gruyter mouton.

Krzysztof Sakowski

\section{FOREIGN OR NATIVE PHRASE ELEMENTS? - COGNITIVE ASPECTS OF THE VERBAL BRACKET IN GERMAN}

\section{(Summary)}

This paper will show why in the German language, such complicated structures as the verbal brace is so prevalent despite obviously presenting a cognitive challenge for foreign language learners. The verbal brace should therefore be studied both from the diachronic and from the contrastive perspectives to show whether it is exclusively a German structure or whether this development has appeared in other Germanic languages too. It is also interesting to consider what it is about the special performance of this structure which has contributed to its success.

Keywords: verbal brace, cognitive linguistics, verbal separability, Ronald W. Langacker, cognitive grammar 